\title{
La imagen de China y la geografía urbana china en Occidente: Reflexiones desde la península Ibérica
}

\author{
Rubén C. Lois González \\ M. ${ }^{a}$ José Piñeira Mantiñán \\ Universidade de Santiago de Compostela. Departamento de Xeografía \\ rubencamilo.lois@usc.es \\ mariajose.pineira@usc.es
}

Recepción: junio de 2016

Aceptación: noviembre de 2016

\section{Resumen}

Según Edward Said, a partir de la edad media, la civilización china ha aparecido, a los ojos de los europeos, como ejemplo acabado de lo oriental. Esto implicó una imagen de lejanía, cierto exotismo y un enorme desconocimiento de las claves internas de este extenso territorio, salvo en contadas excepciones donde lo chino significaba novedad (la pólvora, el vestido, la pasta, etc.). Esta imagen, muy arraigada en Occidente, ha sido transferida a la ciencia europea y norteamericana desde el siglo XIX. De hecho, en el presente artículo, se constata la ignorancia que expresan libros muy prestigiosos de historia y teoría geográficas escritos en español, francés o inglés sobre lo realizado en esta nación. No obstante, el gran potencial exhibido por China durante los últimos decenios ha despertado un notable interés en nuestro mundo. Por eso, se evocan dos grandes obras interpretativas de la lógica económica, la cultura y el comportamiento científico chinos publicados recientemente: la de Gloria Davies y la de Giovanni Arrighi. Una atención que, finalmente, ha pasado al mundo de los estudios geográficos y urbanos, como se demuestra en el recuento efectuado sobre la creciente presencia de trabajos referidos a las ciudades y al proceso de urbanización china en las más prestigiosas revistas globales de la materia.

Palabras clave: China; geografía; conocimiento territorial; sistema de ciudades; proceso de urbanización 
Resum. La imatge de la Xina i la geografia urbana xinesa a Occident: Reflexions des de la peninsula Ibèrica

Segons Edward Said, a partir de l'edat mitjana, la civilització xinesa ha aparegut, als ulls dels europeus, com un exemple acabat del món oriental. Això va implicar una imatge de llunyania, cert exotisme i un enorme desconeixement de les claus internes d'aquest extens territori, tret de comptades excepcions on tot el que estava relacionat amb la Xina significava novetat (la pólvora, la roba, la pasta, etc.). Aquesta imatge, molt arrelada a Occident, ha estat transferida a la ciència europea i nord-americana des del segle xix. De fet, al present article, s'hi constata l'enorme desconeixement que expressen llibres molt prestigiosos d'història i de teoria geogràfiques escrits en espanyol, francès o anglès sobre tot el que s'ha realitzat en aquesta nació. Tanmateix, l'enorme potencial desplegat per la Xina durant els darrers decennis ha despertat un gran interès al nostre món. Per això, s'evoquen dues grans obres interpretatives de la lògica econòmica, la cultura i el comportament científic xinesos publicats recentment: la de Gloria Davies i la de Gianni Arrighi. Una atenció que finalment ha passat al món dels estudis geogràfics i urbans, com es demostra en el recompte efectuat sobre la creixent presència de treballs referits a les ciutats i al procés d'urbanització xinesa en les més prestigioses revistes globals de la matèria.

Paraules clau: Xina; geografia; coneixement territorial; sistema de ciutats; procés d'urbanització

\section{Résumé. L'image de la Chine et de la géographie urbaine de la Chine dans le monde occidental: Réflexions depuis la Péninsule Ibérique}

Selon Edward Said, depuis le moyen-âge, la civilisation chinoise est apparue, aux yeux des Européens, comme un parfait exemple de l'Orient. Il s'agissait une image d'éloignement, d'un certain exotisme et d'une énorme ignorance des clés internes de ce vaste territoire, sauf à rares exceptions où les produits chinois évoquaient la nouveauté (la poudre à canon, la robe, les pâtes, etc.). Cette image profondément enracinée dans le monde occidental a été transférée à la science européenne et américaine depuis le XIxe siècle. En fait, cet article souligne l'ignorance exprimée dans des livres très prestigieux sur d'histoire et la théorie géographiques écrits en espagnol, français ou anglais sur ce qui ce faisait en Chine. Cependant, le grand potentiel affiché par la Chine ces dernières décennies a suscité un intérêt notable dans notre monde. C'est pourquoi nous évoquons deux grandes œuvres interprétatives de la logique économique, de la culture et du comportement scientifique chinois publiés récemment : ceux de Gloria Davies et de Gianni Arrighi, une attention qui s'est finalement transférée au monde des études géographiques et urbaines, comme l'indique le décompte effectué sur la présence croissante de travaux se référant aux villes et au processus d'urbanisation chinois dans les plus prestigieuses revues mondiales de la matière.

Mots-clés: La Chine, géographie, connaissance territoriale, système des villes, le processus d'urbanisation

\section{Abstract. The image of China and Chinese urban geography in the Western World: Reflections from the Iberian Peninsula}

Following Edward Said, since the Middle Ages, Chinese civilization has appeared, to the eyes of Europeans, as a perfect example of the Oriental. This image evoked remoteness and a certain exoticism, and implied a lack of knowledge about the internal keys of this vast territory, except where Chineseness entailed novelty (gunpowder, clothing, pasta, etc.). This deeply rooted image in the West has been transferred to European and American science 
since the nineteenth century. This article verifies the enormous ignorance reflected in prestigious history and geographical theory books written in Spanish, French and English on the nation's achievements. In our world, however, the tremendous potential exhibited by China over the last decades has aroused great interest. In this line, two recently published interpretative works by Gloria Davies and Gianni Arrighi about Chinese economic logic, culture and scientific behaviour are discussed. The field of geography and urban studies have also come to focus attention on these issues, as demonstrated in the growing number of works related to cities of China and the Chinese urbanization process in leading international journals on the subject.

Keywords: China; geography; territorial knowledge; system of cities; urbanization process

\section{Sumario}

1. La imagen de China y la geografía del país desde un conjunto de obras europeas de referencia
2. Los estudios urbanos chinos en

las principales revistas internacionales:

Una aproximación bibliométrica

3. El contenido de este número

Referencias bibliográficas

Desde cualquier punto de vista que se adopte, las magnitudes de la República Popular China en los comienzos del siglo XXI son impresionantes. Estamos ante el país más poblado de la Tierra, con 1.357.000 millones de habitantes en 2013; la segunda economía tras Estados Unidos, que mantiene un crecimiento del $7 \%$ anual (cifra que, si se compara con la del período precedente, los medios occidentales califican habitualmente como parón en su ritmo), y contabiliza la relación más amplia de grandes ciudades del planeta a mucha diferencia de India, su vecino asiático, que se situaría en segundo lugar. De hecho, se estima que posee 20 de las 100 mayores aglomeraciones urbanas del mundo, siempre con poblaciones superiores a los cuatro millones de habitantes.

Frente a la magnitud de estos datos correspondientes a la nueva potencia mundial de la presente centuria, si nos fijamos en nuestro conocimiento sobre la misma, nos sorprenderíamos de su carácter enormemente limitado. Es cierto que la República Popular China se localiza fuera de Occidente y que está muy alejada de nuestro lugar de residencia, pero resulta pasmosa la escasa atención que se presta en la parte del mundo donde nos incluimos a los estudios académicos chinos, más en concreto, a los referidos a ciencias sociales, a geografía y a urbanismo. Solo se reconocen los grandes acontecimientos del país, y el resto de referencias no pasan de ser fragmentarias, puntuales y cargadas de tópicos. Una mínima reflexión sobre este asunto nos muestra hasta qué punto el eurocentrismo, la idea de que Occidente todavía rige el curso y las principales ideas del mundo, está arraigado en nuestro comportamiento.

Frente a este panorama general tan negativo, solo algunos autores influyentes del pensamiento europeo y/o norteamericano han prestado una profunda 
atención al gigante asiático. Entre ellos, sin duda grandes pensadores que, como Jürgen Habermas, Jacques Derrida, Slavo Zizek o Frederick Jameson, han visitado el país para exponer sus ideas e intercambiar opiniones en sus medios académicos e intelectuales (Zhao, 2009). Hace unos pocos años, este interés comenzó a reflejarse en nuestro entorno académico inmediato de la geografía española en forma de breve contribución (Gómez Mendoza, 2013) o se ha integrado en teorías explicativas generales del pensamiento crítico actual (Sassen, 2015). En un plano más concreto, nuestro seguimiento de la popular publicación del pensamiento británico crítico, New Left Review, nos ha permitido identificar a dos autores fundamentales que sí se han preocupado por la cuestión china desde otras tantas perspectivas diferentes y que nos aportan interesantes visiones estructurantes sobre las que vamos a trabajar. La primera es Gloria Davies, autora de un completo libro sobre la intelectualidad china contemporánea que fue editado en Harvard (Davies, 2007), y el segundo es Giovanni Arrighi, economista, que, tras definir el siglo xx como el periodo de la hegemonía norteamericana (Arrighi, 2007), escribió Adam Smith en Pekin, donde se analizan en profundidad las características del desarrollo reciente y del éxito de la que se convertirá en la potencia hegemónica del siglo XxI (Arrighi, 2007).

La obra de G. Davies repasa la tradición intelectual china a lo largo de la historia, jugando frecuentemente con conceptos clásicos en los estudios culturales, como tradicionalismo y cosmopolitismo o liberalismo y neoizquierdismo, a los que añade oposiciones específicamente chinas, como neoconfucionismo y neoiluminismo (Davies, 2007; Zhao, 2009). De sus páginas, se deduce la existencia de una particular cosmovisión de los intelectuales chinos sobre el mundo, encerrada sobre sí misma y deudora de épocas históricas no muy lejanas, en las que los chinos se consideraban la única civilización existente en el planeta. Una situación de aislamiento respecto al exterior, adoptada por diferentes dinastías imperiales, que incluso ha animado teorías no contrastadas sobre el posible descubrimiento chino de América antes de 1492 (Menzies, 2003), que concuerdan con las tradicionales interpretaciones de la historiografía francesa sobre la muy superior capacidad tecnológica de esta civilización oriental respecto a la europea durante todo el siglo xv (Chaunu, 1972). Volviendo a G. Davies, diremos que nos interesan dos afirmaciones de su trabajo: la primera, que los intelectuales chinos asumen las principales corrientes e ideas del pensamiento occidental, pero adaptándolas a su propio contexto. Un contexto determinado por una obligación moral que determina anteponer la mejora y el éxito de China a la abstracción de las aventuras filosóficas de los teóricos occidentales (Davies, 2007). Por eso, aunque H. Zhao defiende el enorme interés de los pensadores chinos por estar al día de las últimas tendencias de la teoría social y filosófica, afirma que el país podría seguir siendo durante algún tiempo un enano en el campo de la investigación crítica, aunque esté destinado a convertirse en la mayor economía del mundo y en un gigante político en el juego global del poder (Zhao, 2009). La segunda opinión de Davies consiste en reivindicar la apertura de la ciencia y del pensamiento chino para corregir su extremada introversión. De hecho, su obra subraya la importancia de las 
trayectorias de estudiantes y profesores chinos en el extranjero (normalmente, en instituciones de expresión anglófona), el afán de internacionalización de sus universidades y las profundas transformaciones que se están operando en la realidad del país (Davies, 2007).

Por lo que se refiere a la obra de G. Arrighi (Arrighi, 2007), este influyente autor se propone explicar las causas estructurales del éxito económico chino en los últimos decenios del siglo xx y su conversión en potencia hegemónica en un proceso ya iniciado (Andreas, 2009). Para este fin, Arrighi utiliza un esquema interpretativo muy arraigado en la historia y en la crítica económica occidental, consistente en suponer que un país se convierte en paradigma del éxito en un período dado y que, por esta razón, pasa a dominar y/o a influir en el resto del mundo. Según esta lectura, el Reino Unido sería la nación exitosa de la industrialización y el capitalismo clásicos del siglo xIx; Estados Unidos, del $\mathrm{xx}, \mathrm{y}$, en el presente siglo XXI, este papel corresponderá a la República Popular China (Arrighi, 2007). Sin duda, este esquema interpretativo es deudor del formulado años antes por Inmanuel Wallerstein en su acercamiento a la «economía-mundo» (Wallerstein, 1979-1998). A lo largo de más de 400 páginas, G. Arrighi realiza un repaso de los grandes pensadores de la sociedad y de la economía contemporáneas - Karl Marx, Adam Smith y, de forma menos profunda, Joseph Alois Schumpeter- para explicar las ondas largas del desarrollo capitalista, sus fuerzas motrices y la cuestión de la hegemonía mundial encarnada por una nación o por una región del planeta. De hecho, y esta es una aportación profundamente original del libro, asocia la teoría de Marx a lo que sucedió en el siglo pasado en Detroit, y a Smith, a la actualidad en Pekín (Arrighi, 2007), argumentando así, en paralelo, otras reflexiones de M. Berman o D. Harvey, que emplean la asociación de la ciudad (innovadora, líder) con un momento de la historia de la civilización (Berman, 1982; Harvey, 2003). En una segunda parte, aborda el tema de la turbulencia global, de aquellas dinámicas sociales y económicas que explican el ascenso y la crisis de un determinado modelo económico en el marco general del capitalismo globalizado. El tercer capítulo se titula «El desmoronamiento de la hegemonía» y, en él, se analizan las razones generales por las que una potencia comienza a declinar y es sustituida por otra, como puede estar aconteciendo entre Estados Unidos y la República Popular China. Finalmente, en la última parte del texto, se aventura el surgimiento de una nueva era asiática, que definirá los principales trazos y la hegemonía del siguiente período de desarrollo capitalista que se aprecia en los decenios centrales del siglo xxI (Arrighi, 2007).

Con respecto a las principales ideas sobre las causas del éxito chino que se apuntan, la inteligente lectura de J. Andreas en New Left Review nos facilita la comprensión de las tesis de Arrighi (Andreas, 2009), puesto que se parte del comentario que el autor realiza de la China anterior y posterior a la Revolución de 1949. En un apartado que se titula "Marx y Mao", establece una relación entre los dos pensadores, al considerar que el marco teórico elaborado por Marx permitiría entender el funcionamiento de la realidad china de la primera mitad del siglo xx y sus contradicciones, una interpretación básica para que Mao 
pudiese culminar su victoria revolucionaria (Andreas, 2009). Todo el período maoísta supuso restringir severamente la enorme tradición mercantil de esta nación oriental e implicó la colectivización de los medios de producción, vinculándolos directamente al factor trabajo. Una interpretación marxista ortodoxa del momento, que, con resultados desiguales, propició la transformación social y económica del país (Andreas, 2009). El periodo post-Mao extendido entre 1976 y 1992 se define como una economía de mercado no capitalista, como consecuencia de las reformas impulsadas por el equipo de Deng Xiaoping. La etapa se inicia con la posibilidad de introducir la iniciativa privada en una economía todavía muy dependiente del sector público. El sector privado desarrollado en las ciudades y surgido en los márgenes de las empresas estatales permitió un auge (o una recuperación) notable del mercado. Así, las urbes se encaminaron por una senda de modernización productiva, con la coexistencia de lo público y lo privado, mientras los cambios en el mundo rural eran mucho más limitados, puesto que se centraban en la descolectivización de la tierra en 1984 y en el retorno de su propiedad al núcleo familiar. Se ahondaron los contrastes entre la ciudad y el campo (origen de los masivos movimientos ilegales de población del presente, que están en el trasfondo de los artículos incluidos en este número), al tiempo que se decidía impulsar la economía capitalista plena a través de la creación de zonas económicas especiales, con abundante entrada de inversiones hongkonesas y de Taiwan (Andreas, 2009).

Desde 1992, se radicalizan las reformas promercado y se accede a una fase de privatizaciones masivas. Se eliminan las restricciones a la iniciativa particular, el país se abre al capital extranjero (fundamentalmente, asiático) y surge el grupo de los xiabai, cuadros y profesionales procedentes del partido que deciden adentrarse en la experiencia empresarial, con procesos de enriquecimiento rápido, normalmente asociado a elevados niveles de corrupción (una de las lacras de la China actual). El proceso privatizador de esta época se tradujo en que, frente a un $82 \%$ de empleo dependiente del sector publico en 1991, se pasase al 27\% en 2005 (Andreas, 2009). En todo este período reciente, el Estado se ha reservado el control de sectores considerados estratégicos para la economía (el campo bancario, el petrolífero, el del acero, el eléctrico, el de las telecomunicaciones y el de la producción armamentística), si bien sus compañías se han sometido a un modelo estricto de organización empresarial a partir de la existencia de consejos de administración que las dirigen, de los objetivos de consecución del máximo beneficio, del aumento de la productividad y de la flexibilidad laboral (que ha implicado por primera vez el despido de trabajadores menos rentables y su sustitución por otros más jóvenes y competitivos) (Andreas, 2009). Este hecho permite concluir a G. Arrighi que, en China, se ha establecido ya una economía capitalista; eso sí, con una cuota de control del aparato estatal y un sector agrario que todavía descansa en el trabajo de cientos de millones de familias campesinas dueñas de sus tierras (si bien se observa una creciente penetración de grandes corporaciones privadas en el mundo rural en lo que se refiere a los ámbitos de la logística, del comercio o de la transformación de los rendimientos obtenidos). Una consecuencia lógica 
de estos cambios operados desde 1992 es el avance, hasta niveles escasamente aceptables, de la polarización social. Tanto G. Arrighi como J. Andreas aportan datos muy llamativos al respecto: la República Popular China contabilizaba, en 2007, un total de 106 personas con un patrimonio de más de 1.000 millones de dólares. Esto es, se situaba en segundo lugar mundial, por detrás de Estados Unidos, en la clasificación de multimillonarios. Además, los cálculos sobre desigualdad de renta de estas fechas mostraban al país asiático como el que contabilizaba un mayor desequilibrio entre ricos y pobres, solo por detrás de Brasil y Sudáfrica y por delante de Estados Unidos (Arrighi, 2007; Andreas, 2009). En los tres artículos que se presentan en este número, se analizan los efectos de dicha polarización y de dicha exclusión de índole socioeconómica en las grandes metrópolis chinas, pero este fenómeno urbano reciente no puede ocultar que, todavía hoy, las principales desigualdades en la potencia asiática persisten entre un mundo urbano competitivo y más rico y uno rural postergado y bastante pobre (Andreas, 2009). El régimen ha optado por impulsar un sistema urbano fuerte y cohesionado, por lo que el espectacular crecimiento de las ciudades más importantes constituye un componente fundamental de la evolución reciente de China.

Como se tuvo ocasión de adelantar, la tesis de G. Arrighi insiste en la existencia de una senda asiático-oriental distinta de desarrollo capitalista que, liderada por la República Popular China, introduce componentes autónomos y novedosos en este tipo de producción (Arrighi, 2007). Así, el principal elemento distintivo del modelo chino sería un fuerte desarrollo de la economía de mercado, de las empresas capitalistas, pero sometidas siempre al control del Estado y del partido. Es en este punto donde las interpretaciones de G. Arrighi y de J. Andreas discrepan, pues si el primero estima que, siguiendo a Adam Smith, el Gobierno chino somete al sector privado a un fuerte control para garantizar un sistema de competencia perfecta que estimule el crecimiento (Arrighi, 2007); para J. Andreas, las interconexiones entre capital y Estado son mucho mayores y contaminan la acción de este último (Andreas, 2009). No obstante, ambos coinciden en destacar el papel del mundo rural como sostenedor del éxito chino, combinado con una idea nacional muy arraigada, y ya mantenida por G. Davies, de que todos los ciudadanos desean contribuir al progreso de su país. Para G. Arrighi, los impresionantes resultados de China se han cimentado en la conservación relativamente atrasada de un mundo rural que envía su fuerza de trabajo sobrante a las ciudades, donde contribuye de forma muy significativa a impulsar la gran fábrica del mundo en la que se ha convertido esta nación, a unos niveles de bienestar colectivo muy aceptables, gracias a los esfuerzos del Gobierno por asegurar un mínimo derecho a la vivienda, así como una educación y una sanidad de calidad (Arrighi, 2007). En el futuro, según estos autores, la República Popular China podrá liderar el importante cambio asiático-oriental en marcha o mantenerse como un gigante económico mundial, pero todavía situado en la periferia del poder (Arrighi, 2007; Andreas, 2009). A lo largo de los próximos años, se tendrá que observar la evolución de este enorme país y su posible conversión en la primera potencia mundial. 


\section{La imagen de China y la geografía del país desde un conjunto de obras europeas de referencia}

Una vez revisadas las dos interpretaciones contemporáneas que estimamos fundamentales para comprender a la China actual, se tratará de retomar uno de los argumentos iniciales que se han utilizado sobre el gran desconocimiento que manifiesta nuestra disciplina en Europa acerca del país y su geografía. Con este objetivo, se han consultado diez publicaciones muy significativas de la teoría geográfica del continente para comprobar la cantidad y la calidad de las referencias que se realizan sobre la mencionada potencia oriental. Esta búsqueda ha respetado una cierta diversidad interna de escuelas nacionales y así se han analizado: en español, las obras centrales de H. Capel, Gómez Mendoza et al. y J. Ortega Valcárcel sobre teoría e historia de la geografía (Capel, 1981; Gómez Mendoza et al., 1982; Ortega, 2000); en francés, se ha preferido estudiar el contenido de los dos diccionarios más influyentes de los últimos decenios, el de R. Brunet et al. (1992) y el de J. Levy y M. Lussault (2003); en inglés, también se recurre a diccionarios, el de Oxford y el de R.J. Johnston, D. Gregory y D.M. Smith en su primera edición (Johnston et al., 1987; Mayhew, 1992); en italiano, se ha consultado una obra central de F. Farinelli titulada Geografia (Farinelli, 2003), y, para finalizar, se ha recurrido a dos obras organizadas por capítulos de distinta autoría, una claramente francófona (Bailly et al., 1984) y otra que procura equilibrar las tradiciones francesa y anglófona, si bien ha sido editada en inglés (Benko y Strohmayer, 2004). Con respecto a este análisis, cabe matizar que, aunque se debe considerar eminentemente europea, la trayectoria de autores como D. Gregory se ha visto marcada posteriormente por un traslado profesional a Canadá, mientras que son varios los estudiosos norteamericanos que participan en el último libro citado (N. Entrikin, A. Scott, P. Gould, etc.). Por lo tanto, nos encontramos ante interpretaciones occidentales clásicas y autorizadas, todavía muy influidas por el eurocentrismo, si bien recientes aproximaciones al tema de las ciudades y la planificación urbana en la República Popular China ya reflejan un conocimiento mucho mejor de la realidad de este país, llamado a convertirse en fundamental en un plazo relativamente breve (Friedmann, 2011).

En el caso de las tres contribuciones españolas, al menos dos de ellas expresan una cierta curiosidad por el pensamiento geográfico asiático en general y chino en particular a lo largo de la historia. Así, en Filosofía y ciencia en la geografía contemporánea, cuando se inicia un repaso exhaustivo del proceso de institucionalización geográfica en diferentes regiones del mundo, se aporta una relación y unas citas genéricas acerca de sociedades geográficas y de revistas de la disciplina contabilizadas en Asia en el siglo xIx y comienzos del xx (Capel, 1981). Más amplia es la referencia a la China imperial realizada por J. Ortega Valcárcel en las páginas 28, 31 y 32 de su extensa obra (Ortega, 2000). Este autor hace una alusión al hecho de que todas las civilizaciones, por primitivas que sean, disponen de conceptos y de procedimientos de orientación y localización siguiendo, por ejemplo, los puntos cardinales. Estas prácticas ya aparecían 
en la China de la antigüedad, donde, como apuntan autores japoneses, «el Este parece haber sido originariamente la orientación primordial» (Ortega, 2000). Más adelante, el libro Los horizontes de la geografía introduce una argumentación relativamente repetida en los libros de nuestra disciplina, consistente en señalar que la civilización china se ha preocupado tradicionalmente por la acumulación de conocimientos de carácter espacial, una preocupación impulsada desde el Estado y que alcanzó una notable perfección gráfica dirigida a los objetivos del control, la administración y la gestión territoriales. El primer documento de este tipo correspondería al Imperio Chou y fue elaborado en el siglo $\mathrm{v}$ antes de nuestra era con una finalidad eminentemente tributaria. Conforme se fue consolidando este tipo de informes, se avanzó en la realización de topografías locales que incluían caracteres físicos, relación de recursos y otros componentes del territorio (Ortega, 2000). Otro conjunto de obras de cronología similar, puesto que se inician en el siglo IV aC, serían los libros de viajes e itinerarios, de contenido parecido a los que se cultivaron también en Occidente y en el mundo islámico (Ortega, 2000). La continuidad del Estado a lo largo de los siglos facilitó el mantenimiento de este tipo de saberes del espacio, que tuvieron en China un desarrollo más coherente que en otras regiones del mundo. De ahí que esta civilización exprese un dominio superior de la representación cartográfica, que alcanza uno de sus momentos culminantes con la realización del $Y u$ Chi Thu en piedra a principios del siglo XII. En este y en otros mapas o atlas realizados, destaca el enorme detalle con el que se llegan a representar la línea de costa; los trazados fluviales; los núcleos de población, entre ellos las ciudades, y las unidades catastrales. En este aspecto, se aprecia una diferencia con el mundo occidental, ya que en China se describe lo propio, lo correspondiente al Imperio con suma minuciosidad y precisión, a diferencia del territorio restante, que apenas interesa (Ortega, 2000). A este respecto, la geografía de esta civilización se diferenciaría de la occidental, siempre más preocupada por la forma y las dimensiones del mundo, ya que, en el Mediterráneo y en Europa, la movilidad, los cambios históricos y las migraciones siempre han tenido una mayor importancia. Para concluir este repaso de obras teóricas españolas, cabe precisar que, en El pensamiento geográfico, de los más de 400 autores citados, no aparece ninguno chino, ni siquiera referencias a la cultura territorial de este importante país (Gómez Mendoza et al., 1982). No obstante, una de sus autoras, J. Gómez Mendoza, sí se ha fijado, en épocas recientes, en la urbanización china con un breve, pero bien fundamentado, texto que aporta un conjunto de claves a la dinámica de crecimiento de las ciudades de esta gran potencia oriental (Gómez Mendoza, 2013).

Aunque la recopilación de informaciones sobre la importancia de la tradición geográfica china puede parecernos débil en el caso español, el panorama no mejora en los diccionarios franceses y británicos consultados, que, además, suelen mostrar muchos más prejuicios culturales. Así, en el escrito desde la Maison de la Géographie de Montpellier, realizado por un equipo encabezado por R. Brunet, se han podido documentar hasta siete palabras que introducen una alusión a la cultura y a la civilización chinas (Brunet et al., 1992). Cuatro 
de estas alusiones son bastante banales, como chino (chinois), dragón, Gobi ("desierto más o menos pedregoso», se nos dice) y Oriente ("donde nace el Sol»). Sorprende la relativa ligereza con que toda la obra trata lo oriental, lo chino. En cualquier caso, en geografía, definida en tanto ciencia, se matiza que, en mongol, se la denomina incluso como la ley de las comarcas y se señala que la cuestión fundamental que esta civilización se pregunta es "¿donde?», lo que enlazaría con las consideraciones mucho más matizadas sobre las descripciones territoriales de esta civilización que se realizan en la obra de Ortega Valcárcel. Esta apreciación sobre el legado tradicional de la geografía china se repite en la explicación de Histoire de la Géographie, donde se apunta: «los chinos, hace más de 3.000 años, poseían una información geográfica que era poco crítica sobre su propio mundo y estaba poco atenta a aquello que le rodeaba», disponían "de una visión muy profunda de su Imperio, de sus comunicaciones y ciudades, y mapas» (Brunet, Ferras y Théry, 1992). Estamos ante una información muy generalista y dominada por prejuicios culturales que, por último, se manifiestan en la explicación del término oriental: «Etnotipo: alguien [...] cruel, que no merece confianza; esta afirmación se remonta a Marco Polo [...], sin duda por lo cruel a las incursiones de Tártaros y Mongoles. Igualmente, paralizado y poco dado al cambio según Montesquieu» (Brunet, Ferras y Théry, 1992).

En el diccionario de Levy y Lussault, la escasez de referencias se mantiene, así como el enorme peso del eurocentrismo de una buena parte de la geografía francesa. Lo chino (o lo oriental) se recoge en tan solo cuatro palabras: ecúmene, imperio, frontera y geografía (Levy y Lussault, 2003). En la primera, se vuelve al ensimismamiento de la civilización que nos ocupa, cuando se alude a que China se denominaba a sí misma "el país del centro». La explicación se basa en el significado de "Imperio mundo» que se aplica a la civilización china, aunque luego también se apunta al hecho de que algunos de ellos se han convertido en naciones poderosas, como Alemania y China. La penúltima referencia es a frontera, y se vuelve a recurrir a un tópico, pues «la dificultad en regular los contenciosos fronterizos en el mar (como en el mar de China del Sur entre China, Vietnam, Taiwan y Filipinas) explica de hecho el carácter muy particular de la línea fronteriza» (Levy y Lussault, 2003). Finalmente, en geografía, se alude a las prácticas de exploración y descripción sistemáticas del territorio cultivadas por la China imperial.

Dentro del grupo de obras geográficas relevantes que hemos analizado, quizás el enfoque más desconsiderado, y descorazonador, sobre China y su civilización se encuentra en el Diccionario de Geografía, de Oxford. Estamos ante una edición de bolsillo con menos pretensiones, pero avalada por una de las principales instituciones académicas del mundo (Mayhew, 1992). En la misma, la situación de la China de fines del siglo xx se despacha en tres definiciones: antinatalista, granja colectiva y factor de arrastre. Para antinatalista, se insiste en explicar la política del hijo único adoptada en este país como coercitiva desde el Estado y que ha acarreado el infanticidio de niñas en distintas provincias, hecho demostrado estadísticamente (Mayhew, 1992). En referencia a granja colectiva, se debe tener en cuenta que el diccionario se elaboró en plena 
perestroika, con lo cual la tendencia a la culpabilización se dirigía directamente al régimen chino. Así, este modelo combina las propiedades individuales de los granjeros con otras que pertenecen al Estado. Se continúa aclarando que la parte colectiva es la responsable de pagar a los empleados y también la de comprar los inputs. Las autoridades fijan los objetivos de producción desde un modelo de planificación centralizado, en una lógica que se comienza a colapsar en Europa del Este y en la URSS, y que es desplazada por formas privadas de trabajo en China (Mayhew, 1992). Como se puede apreciar, no encontramos ninguna referencia concreta a las reformas económicas iniciadas en este país desde 1976 ni, de forma particular, a las leyes de descolectivización de la tierra de 1984. Por último, en esta lectura marcada por prejuicios, en lo que se refiere a factor de arrastre, se alude al elemento positivo que genera una concentración hacia una localidad donde la gente migra, porque recibe estímulos materiales, como es el caso de Hong Kong (Mayhew, 1992). Además, y como colofón final, el diccionario nos obsequia con una serie de datos estadísticos por países, y después de su revisión, se comprueba que no está China.

Si pasamos al muy popular Diccionario de Geografía Humana dirigido por R.J. Johnston, D. Gregory y D.M. Smith, en su primera edición (Johnston, Gregory y Smith, 1987), se han localizado cuatro voces donde aparece tratado lo chino o lo oriental. En la primera, ciudad franca (o puerto franco), se nos indica que "Hong Kong, importante ejemplo actual, funciona bien porque forma parte de un microestado en buena medida aislado de la República Popular China» (Johnston et al., 1987). Por su parte, en colonialismo, se nos recuerda lo siguiente:

[...] el creciente dominio de Gran Bretaña como centro de poder económico a comienzos del siglo XIx y el consiguiente auge del laissez-faire en las relaciones económicas internacionales hizo que ya no fuese tan necesario el colonialismo mercantilista que subyacía en la ocupación del continente americano, del Caribe, de algunas zonas de África, India y el Lejano Oriente. No obstante, fue en esta época cuando China sufrió los embates del colonialismo, sin ser sometida a un dominio directo. (Johnston et al., 1987)

Acerca de economía del desarrollo, se indica que «los estadios de crecimiento no reflejan exactamente la realidad, dado que podrían aplicarse a cualquier lugar y a cualquier momento, a China y a Brasil, a la URSS y a EE. UU.», en referencia crítica a la interpretación de W. Rostow (Johnston et al., 1987). Finalmente, la explicación de la palabra soberanía nos recuerda que, en la práctica, los estados suelen crecer con más frecuencia de lo que se cree mediante la conquista, como demostraron los chinos en el Tíbet. Se continúa diciendo, sin aflojar el tono severo de los comentarios hacia el gigante de Oriente, que, en general:

[...] la soberanía es el derecho nacional más universalmente respetado en toda la comunidad mundial y cualquier violación se condena siempre unánimemente. Sin embargo, bajo ciertas circunstancias, puede ser parcialmente revocado, 
como en el caso de desastres naturales [...]. Incluso en estas circunstancias externas, si un estado es lo suficientemente perseverante, puede llegar a evitar injerencias o ayudas [...] como demostró China cuando rehusó toda ayuda extranjera para enfrentarse al gigantesco terremoto en la provincia de Hopei que pudo haber causado la muerte de 700.000 personas en 1976. (Johnston et al., 1987)

Junto con la obra de J. Ortega Valcárcel, el otro texto consultado que sí ofrece una imagen sugerente de la civilización china es Geografia, de F. Farinelli (2003). En el mismo, de fuerte carácter evocativo, la narración sobre los temas relevantes de la teoría espacial y territorial se estructura en pequeños textos independientes de dos a cuatro páginas, que permiten ir saltando de una cuestión a otra próxima. Según nuestra consulta, a lo chino se le dedican parcialmente cinco textos. El primero, el número 6 de la obra, se titula «La duración del Mundo: Marco Polo». De hecho, este viajero italiano empleó tres años y medio en regresar a causa de la nieve, la lluvia y las grandes inundaciones que tuvo que padecer. En el año 1300, se estimaba que la duración normal del viaje desde Crimea hasta China era de nueve meses. Existía una alternativa rápida para afrontar este trayecto: el yan, el sistema postal del Imperio mongol, basado en una red de estaciones con mensajeros distantes 25 millas una de la otra. Este modelo es descrito por Marco Polo, que insiste en la linealidad, rapidez y equivalencia de las partes (Farinelli, 2003). Volviendo a la idea de civilización ensimismada, F. Farinelli nos cuenta, en su texto 45, "Lo que se mueve y lo que está cerrado». Se refiere a que, en un texto de la sabiduría china escrito por el padre del taoísmo, se describe al fin la felicidad de un país ideal: donde nunca más se utilizan los instrumentos de trabajo, no se emplean medios de transporte, donde las personas no emigran, y donde existen pueblos distribuidos de un modo tal que, si se oye cantar al gallo o ladrar al perro, ya no se necesita visitar al vecino (Farinelli, 2003). Frente a esta visión, el geógrafo italiano contrapone la historia de Ulises, que es llamado a huir, a viajar y a descubrir nuevos mundos, y que, cuando regresa a su hogar, conserva el recuerdo, el conocimiento obtenido en su largo periplo (Farinelli, 2003). En un plano de reflexión contemporánea, el texto 49, «La rana, el estanque, la tierra», nos recuerda que la mitad de los habitantes de la Tierra se concentra en una sexta parte de las áreas emergidas. Y continúa:

[...] en realidad, en China y en Europa la densidad varía, y mucho, a medida que se pasa de la costa al interior. Si por China se entiende de hecho la China histórica de las 18 provincias orientales, sobre una superficie de 4 millones de $\mathrm{km}^{2}$ [...], la concentración euroasiática resulta casi el doble, más de 2.500 millones de personas, casi la mitad de los seres humanos en poco más de la doceava parte de las tierras emergidas. (Farinelli, 2003)

Esta reflexión se mantiene en el texto 52, "Hombres (mujeres) y clima», donde se señala que, en la antigüedad, la principal dorsal de poblamiento se alargaría en la línea de clima subtropical del hemisferio septentrional, del Medi- 
terráneo a Mesopotamia, la India y China, en un casi perfecto acuerdo con la teoría del clima (Farinelli, 2003). Por último, en el texto 95, denominado «La ciudad global», se nos dice:

[...] las ciudades globales no son necesariamente las más grandes del planeta. Cuando las primeras fueron reconocidas como tales, según la clasificación de la ONU, las ciudades que superaban los 10 millones de habitantes eran 13: Tokio, con 25 millones; Sao Paulo, con 20; Nueva York y Ciudad de México, con 15, y luego Shanghai, Bombay, Los Ángeles, Buenos Aires, Seúl, Pekín, Río de Janeiro, Calcuta y Osaka [...]. Como se deduce de una primera comparación, las ciudades europeas y norteamericanas controlan, aunque el grupo de asiáticas y latinoamericanas se engrandece. (Farinelli, 2003)

Sin lugar a dudas, F. Farinelli se encuentra entre los geógrafos occidentales más sensibles y preocupados por el pensamiento y las prácticas espaciales de otras civilizaciones, en especial las asiáticas (Lladó, 2013). Su interés central por la importancia del mapa en la estructuración del conocimiento desde la antigüedad y sus búsquedas teóricas sobre las diferentes razones cartográficas que se han formalizado en la historia de la humanidad justifican esa atención a China y a su civilización en diversas obras. Otro autor que se ha dedicado con intensidad al estudio de la geografía histórica de Asia y al estudio geográfico de las naciones orientales es el portugués F. Roque de Oliveira, que ha abordado esta cuestión en diferentes contribuciones recientes (Roque de Oliveira, 2003, 2007).

En la obra clásica de los geógrafos francófonos titulada Les concepts de la Géographie Humaine, dirigida por A. Bailly, solo aparece una única referencia indirecta a lo chino (Bailly et al., 1984). La misma corresponde a la autoría de P. Claval en su capítulo sobre la geografía social y cultural. Este autor nos indica "pero el mundo se convierte en terriblemente blando y monótono en el momento en el que los bloques de vivienda social o equivalentes son los mismos en Hong Kong, Moscú, París, Haifa, Río de Janeiro...» (Bailly et al., 1984). Por lo tanto, volvemos a encontrarnos con la misma ignorancia sobre otras culturas y tradiciones de nuestra disciplina que fue mayoritaria en los decenios finales del siglo xx y que también aquí se concreta en el hecho de que no aparece referencia alguna a ningún autor, obra o tema de interés chino (Bailly et al., 1984).

Un texto colectivo mucho más reciente coordinado por G. Benko y U. Strohmayer hace poco más de diez años muestra un mayor interés por otras culturas y los grupos de geógrafos que han trabajado fuera de Europa y de América del Norte, aunque el tema todavía se aborda de forma insuficiente (Benko y Strohmayer, 2004). Así, en el prefacio de este libro colectivo, se reconoce que es necesario «hacer una autocrítica del anglocentrismo de la obra, de sus limitaciones para aprovechar la bibliografía de indios, japoneses, latinoamericanos y africanos». Como se comprueba, los chinos siguen sin aparecer (Benko y Strohmayer, 2004). Luego, una lectura detenida de los textos nos permite comprobar la existencia de dos referencias a China. La primera, indirecta 
e incluida en las primeras páginas de esta revisión teórica de la disciplina, se refiere a la represión de los científicos estadounidenses acusados de comunistas o filocomunistas en la época del macarthismo. En concreto, se centra en el proceso que sufrió el gran experto en China y Mongolia, O. Latimore, de la Universidad John Hopkins de Baltimore, que, a pesar de no concluir con una demostración de los hechos por los que se le acusaba (simplemente, una serie de comentarios privados realizados a un colega), tuvo grandes problemas para continuar con su carrera académica, en un contexto de miedo generalizado a las purgas en la enseñanza superior de EE. UU. durante la década de 1950 (Benko y Strohmayer, 2004). Más adelante, cuando se trata acerca del capítulo de la geografía histórica, se afirma:

[...] la misma aproximación se realizó en China y en otros países del este de Asia, donde [esta rama de la disciplina] volvió su atención sobre el estudio de los antiguos paisajes de la geografía regional, documentados a través de notables inventarios ambientales, demográficos y administrativos. (Benko y Strohmayer, 2004)

Incluso se incluyen citas de algunos autores orientales, en una tendencia al redescubrimiento lento y progresivo de los análisis territoriales y de la geografía milenaria que proviene de Asia.

Para terminar este amplio apartado, y corroborando la impresión de que el análisis y la planificación territorial chinos van alcanzando un reconocimiento progresivamente mayor en Occidente, se debe destacar la atención que los grandes congresos internacionales de geografía empiezan a prestar a ese país, como lo demuestra el hecho de que el XXXIII Congreso de la UGI se haya celebrado en Beiging en 2016 y que, en las últimas reuniones anuales de la AAG, se dediquen sesiones específicas a la geografía china. Este interés lo refleja de forma magistral el urbanista y planificador J. Friedmann en un artículo reciente publicado en español (Friedmann, 2011). En el mismo, este maestro de geógrafos y especialistas en la ciudad aborda el tema de la participación pública en la planificación urbanística. Aunque, para su exposición, se remonta a sus inicios académicos y aborda referencias a grandes pensadores sobre el tema (de T. Campanella a H. Lefebvre y J. Jacobs), de inmediato y para nuestra sorpresa, se centra en la experiencia china sobre la creación de lugares de reunión, el estudio acerca de la dinámica de la vida urbana cotidiana, la calidad del entorno construido y el papel de los barrios en el complejo sistema urbano de ese país oriental (Friedmann, 2011). Sin duda, lo más importante del texto de J. Friedmann es, de nuevo, su atención por una potencia que será clave para la historia del siglo XXI y por el mundo de macrociudades complejas que estamos obligados a conocer mejor. Cuanta más preocupación muestren los geógrafos urbanos occidentales por la vida y las investigaciones urbanas chinas, mejor reflejarán su entendimiento y su adaptación a un mundo cambiante que es global, policéntrico y que se articula sobre realidades metropolitanas. 


\section{Los estudios urbanos chinos en las principales revistas internacionales: Una aproximación bibliométrica}

La presente reflexión sobre el conocimiento de la geografía y los estudios urbanos chinos en los países occidentales se completa con una rápida revisión de los artículos dedicados a este país en diez de las revistas con mayor impacto mundial (estimado por el JCR), incluidas en los listados de geografía humana y urbanismo. Se trata de las siguientes: Antipode, Landscape \& Urban Planning, Journal of Urban Economics, Cities, Urban Geography, International Journal of Urban \& Regional Research, Environment \& Urbanization, Journal of Planning Literature, Urban Studies y Journal of Urban Affairs. Todas ellas están incluidas en los cuartiles superiores de la relación del JCR, por lo que su atención a lo chino y a las investigaciones sobre la ciudad y las realidades metropolitanas de esta gran nación dibujan un panorama significativo con respecto a la importancia que se le concede en la actualidad. En nuestra aproximación, se ha revisado el conjunto de artículos publicados por dichos medios en los últimos diez años (un total de 9.390 de 2005 a 2015), de los cuales tan solo el 4,1\% se refiere a China (en números absolutos, 390 contribuciones editadas).

En este contexto general de débil presencia de este territorio, que, como ya apuntamos, concentra el mayor número de grandes ciudades y megalópolis del mundo, se aprecia (como también se deducía del análisis de libros y diccionarios) que el interés por lo chino se incrementa conforme nos acercamos a la actualidad: de un $2,4 \%$ a un $2,6 \%$ de textos incluidos en estas revistas en el cuatrienio 2005-2008, se pasa a valores superiores al 6\% en 2014 y 2015. Se asiste, por lo tanto, a un tímido despertar de la curiosidad por la gran potencia oriental, en una dinámica que muestra paralelismos con otros países emergentes (India, Rusia, Tailandia, Brasil, etc.) localizados fuera del mundo occidental. No obstante, la receptividad a lo llegado de China todavía

Tabla 1. Total del número de artículos de cada revista en el período 2005-2015

\begin{tabular}{lrrrrrrrrrrrr}
\hline Revista / Total artículos & 2005 & 2006 & 2007 & 2008 & 2009 & 2010 & 2011 & 2012 & 2013 & 2014 & 2015 & Total \\
\hline Antipode & 96 & 74 & 63 & 83 & 62 & 80 & 101 & 104 & 77 & 84 & 45 & 869 \\
\hline Landscape \& Urban Planning & 113 & 139 & 179 & 135 & 141 & 153 & 208 & 205 & 172 & 202 & 109 & 1.756 \\
\hline Journal of Urban Economics & 61 & 63 & 59 & 99 & 54 & 62 & 40 & 54 & 52 & 61 & 26 & 631 \\
\hline Cities & 63 & 59 & 56 & 47 & 52 & 65 & 66 & 76 & 195 & 119 & 82 & 880 \\
\hline Urban Geography & 51 & 45 & 57 & 58 & 59 & 78 & 76 & 73 & 70 & 76 & 32 & 675 \\
\hline International Journal of Urban & 101 & 90 & 75 & 92 & 109 & 94 & 104 & 118 & 158 & 181 & 27 & 1.149 \\
\& Regional Research & & & & & & & & & & & & \\
\hline Environment \& Urbanization & 29 & 31 & 33 & 33 & 30 & 35 & 36 & 38 & 33 & 36 & 20 & 354 \\
\hline Journal of planning literature & 21 & 15 & 5 & 9 & 10 & 13 & 18 & 24 & 14 & 18 & 8 & 155 \\
\hline Urban Studies & 141 & 138 & 163 & 181 & 194 & 205 & 247 & 247 & 253 & 336 & 185 & 2.290 \\
\hline Journal of Urban Affaires & 47 & 46 & 46 & 50 & 53 & 51 & 48 & 56 & 52 & 124 & 58 & 631 \\
\hline Total & 723 & 700 & 736 & 787 & 764 & 836 & 944 & 995 & 1.076 & 1.237 & 592 & 9.390 \\
\hline
\end{tabular}

Fuente: elaboración propia. 
Tabla 2. Número de artículos sobre ciudades chinas respecto al total de artículos publicados para cada año en el período 2005-2015

\begin{tabular}{lrrrrrrrrrrrrr}
\hline Revista / Total artículos & 2005 & 2006 & 2007 & 2008 & 2009 & 2010 & 2011 & 2012 & 2013 & 2014 & 2015 & Total \\
\hline Antipode & 0 & 0 & 1 & 0 & 3 & 0 & 0 & 0 & 1 & 1 & 0 & 6 \\
\hline Landscape \& Urban Planning & 1 & 8 & 6 & 4 & 4 & 4 & 9 & 5 & 3 & 13 & 8 & 65 \\
\hline Journal of Urban Economics & 0 & 1 & 0 & 2 & 1 & 0 & 0 & 1 & 0 & 1 & 1 & 7 \\
\hline Cities & 2 & 0 & 2 & 3 & 3 & 5 & 9 & 3 & 22 & 16 & 21 & 86 \\
\hline Urban Geography & 7 & 1 & 2 & 5 & 5 & 6 & 5 & 9 & 2 & 2 & 1 & 45 \\
\hline $\begin{array}{l}\text { International Journal of Urban } \\
\text { \& Regional Research }\end{array}$ & 2 & 1 & 2 & 1 & 9 & 5 & 6 & 6 & 1 & 8 & 0 & 41 \\
\hline Environment \& Urbanization & 0 & 3 & 0 & 0 & 0 & 2 & 0 & 2 & 0 & 1 & 2 & 10 \\
\hline Journal of planning literature & 0 & 0 & 0 & 0 & 0 & 0 & 1 & 0 & 0 & 1 & 0 & 2 \\
\hline Urban Studies & 5 & 2 & 6 & 3 & 11 & 10 & 12 & 9 & 11 & 29 & 9 & 107 \\
\hline Journal of Urban Affaires & 2 & 1 & 0 & 2 & 0 & 3 & 1 & 1 & 1 & 3 & 0 & 14 \\
\hline Total & 19 & 17 & 19 & 20 & 36 & 35 & 43 & 36 & 41 & 75 & 42 & 383 \\
\hline Fur
\end{tabular}

Fuente: elaboración propia.

es desigual entre los medios elegidos y depende de factores como el objetivo y la temática general de la revista, además de la composición de su consejo editorial. De hecho, como ejemplo significativo, se puede contraponer la publicación que más atención ha prestado a China recientemente, Cities, con un 9,8\% de sus artículos dedicados a dicho país en los últimos diez años, y la que menos, la radical y no estrictamente especializada en geografía urbana Antipode, con una cifra de tan solo el 0,7\%. La primera establece su objetivo como publicación periódica en la política y el planeamiento urbanos. Posee un comité editorial dominado por los académicos estadounidenses, con representantes de otras regiones del mundo (como la española M. a D. García Ramón) y se preocupa bastante por lo chino. Se puede deducir como impresión (no como tesis completamente contrastada) que Norteamérica comienza a mostrar curiosidad por lo que sucede en la gran potencia oriental (los congresos de la AAG y el artículo de John Friedmann también lo indican) y, en especial, en aquellas temáticas que tienen que ver con los grandes desarrollos y proyectos urbanos recientes, el estudio de los nuevos flagships (o nuevos edificios emblemáticos) de la ciudad, y la morfología y la estructura de las megalópolis. Por su parte, la geografía radical sigue concentrándose en Occidente o en el mundo menos desarrollado, pero tiene dificultades para comprender o recibir novedades desde China. El comité editorial de Antipode sigue estando dominado por un agrupamiento de académicos procedentes de países de habla inglesa (sudafricanos, canadienses, estadounidenses, ingleses, etc.) y expresa una dificultad evidente para comprender qué ha pasado en un tradicional referente del comunismo hoy convertido en un exitoso ejemplo de economía de mercado con un crecimiento espectacular y continuo. Quizás dicha insuficiencia solo sea compensada dentro de este enfoque con la gran aportación de G. Arrighi 
Tabla 3. Porcentaje de artículos sobre ciudades chinas respecto al total de artículos publicados para cada año en el período 2005-2015

\begin{tabular}{|c|c|c|c|c|c|c|c|c|c|c|c|c|}
\hline evista & 2005 & 2006 & 2007 & 2008 & 2009 & 2010 & 2011 & 2012 & 2013 & 2014 & 2015 & Total \\
\hline Antipode & $0,0 \%$ & $0,0 \%$ & $1,6 \%$ & $0,0 \%$ & $4,8 \%$ & $0,0 \%$ & $0,0 \%$ & $0,0 \%$ & $1,3 \%$ & $1,2 \%$ & $0,0 \%$ & $0,7 \%$ \\
\hline Landscape & $0,9 \%$ & $5,8 \%$ & $3,4 \%$ & $3,0 \%$ & $2,8 \%$ & $2,6 \%$ & $4,3 \%$ & $2,4 \%$ & $1,7 \%$ & $6,4 \%$ & $7,3 \%$ & $3,7 \%$ \\
\hline ourn & $0,0 \%$ & $1,6 \%$ & $0,0 \%$ & $2,0 \%$ & $1,9 \%$ & $0,0 \%$ & $0,0 \%$ & $1,9 \%$ & $0,0 \%$ & $1,6 \%$ & $3,8 \%$ & $1,1 \%$ \\
\hline Sities & $3,2 \%$ & $0,0 \%$ & $3,6 \%$ & $6,4 \%$ & $5,8 \%$ & $7,7 \%$ & $13,6 \%$ & $3,9 \%$ & $1,3 \%$ & $13,4 \%$ & $25,6 \%$ & $9,8 \%$ \\
\hline Urban & $13,7 \%$ & $2,2 \%$ & $3,5 \%$ & $8,6 \%$ & $8,5 \%$ & $7,7 \%$ & $6,6 \%$ & $12,3 \%$ & $2,9 \%$ & $2,6 \%$ & $3,1 \%$ & $6,7 \%$ \\
\hline $\begin{array}{l}\text { nternational Journal } \\
\text { \& Regional Researc }\end{array}$ & $2,0 \%$ & $1,1 \%$ & $2,7 \%$ & $\%$ & $3 \%$ & $5,3 \%$ & $\%$ & $5,1 \%$ & $\%$ & $4,4 \%$ & $\%$ & $3,6 \%$ \\
\hline Eviror & $0,0 \%$ & $9,7 \%$ & $0,0 \%$ & $0,0 \%$ & $0,0 \%$ & $5,7 \%$ & $0,0 \%$ & $5,3 \%$ & $0,0 \%$ & $2,8 \%$ & $10,0 \%$ & $2,8 \%$ \\
\hline Journal of plann & $0,0 \%$ & $0,0 \%$ & $0,0 \%$ & $0,0 \%$ & $0,0 \%$ & $0,0 \%$ & $5,6 \%$ & $0,0 \%$ & $0,0 \%$ & $5,6 \%$ & $0,0 \%$ & $1,3 \%$ \\
\hline Urban S & $3,5 \%$ & $1,4 \%$ & $3,7 \%$ & $1,7 \%$ & $5,7 \%$ & $4,9 \%$ & $4,9 \%$ & $3,6 \%$ & $4,3 \%$ & $8,6 \%$ & $4,9 \%$ & $4,7 \%$ \\
\hline Journal & $4,3 \%$ & $2,2 \%$ & $0,0 \%$ & $4,0 \%$ & $0,0 \%$ & $5,9 \%$ & $2,1 \%$ & $1,8 \%$ & $1,9 \%$ & $2,4 \%$ & $0,0 \%$ & $2,2 \%$ \\
\hline Total & $2,6 \%$ & $2,4 \%$ & $2,6 \%$ & $2,5 \%$ & $4,7 \%$ & $4,2 \%$ & $4,6 \%$ & $3,6 \%$ & $3,8 \%$ & $6,1 \%$ & $7,1 \%$ & $4,1 \%$ \\
\hline
\end{tabular}

Fuente: elaboración propia.

ya comentada y con la relativa atención que expresa la también emblemática New Left Review por lo chino.

A partir de estas variaciones detectadas entre las diferentes cabeceras, nos ha interesado realizar una breve aproximación cualitativa a la temática y al contenido de los trabajos publicados en tres de ellas. Publicaciones muy significativas, tanto por su prestigio como por prestar una atención desigual a la ciudad y al mundo urbano chinos. Se trata de Antipode, Urban Studies (con un valor similar a los promedios generales) y Cities (que, desde 2014, se convierte en una revista notablemente atraída por los estudios sobre China, con el 13,4\% y el 25,6\% de sus artículos que tratan dicho asunto en 2014 y 2015, respectivamente).

En Antipode, los seis artículos de temática china publicados entre 2005 y 2015 se dedican a analizar los acontecimientos urbanos y territoriales más relevantes acontecidos en dicho país en épocas recientes. De manera especial, en cinco de los seis trabajos, se abordan las consecuencias de las transformaciones capitalistas registradas en el proceso de expansión de la ciudad china. Se refieren a los fenómenos de acumulación primaria en la economía urbana, la emergencia de un modelo de ciudad neoliberal o la pérdida de interés estético y paisajístico de las nuevas áreas residenciales destinadas a la clase media. En un par de artículos, se introducen temas de geografía social urbana de contenido similar a los incluidos en este número de Documents d'Anàlisi Geogràfica y referidos al derecho a la ciudad de los grupos excluidos y marginados, así como a la reestructuración de las zonas comerciales en la ciudad de Guangzhou. Una última contribución critica la visión reduccionista, impulsada por los medios neoconservadores, acerca del conflicto del Tíbet generado por la ocupación china. En definitiva, una temática pegada a la actualidad, cultivada 
desde un punto de vista crítico y argumentativo, pero que todavía expresa una débil curiosidad del pensamiento geográfico radical por lo que sucede en el gran gigante asiático.

En referencia a Urban Studies, la abundancia de textos publicados por dicha revista justifica que dedique un total de 107 artículos a China. En un análisis temático de su contenido, nos encontramos con tres cuestiones fundamentales abordadas: el acceso a la vivienda, la segregación socioespacial y las migraciones urbanas, con 40 artículos; el crecimiento y la modernización infraestructural de las ciudades chinas, con 22, y el mercado y el precio del suelo, con 15 . A cierta distancia, encontramos los estudios sobre el sistema urbano, que contabilizan 9 ejemplos, y sobre los problemas ambientales de la ciudad, con 4. No es posible clasificar los restantes trabajos (un total de 19). Por lo general, y este número de Documents d'Anàlisi Geogràfica en español así lo recoge, la geografía urbana china se preocupa de forma mayoritaria por el mosaico social, las diferencias de renta, étnicas y de estatus de las poblaciones que ocupan, más o menos mezcladas, los enormes espacios urbanos y periurbanos de las metrópolis chinas. El enfoque crítico de estos artículos es importante; en cierta medida, la aproximación a los problemas residenciales y de desigualdad social en el interior de la urbe se plantean con una vocación de analizar disfunciones que deben ser conocidas, publicitadas y, sin duda, solucionadas. Resulta evidente el papel activo de la academia en el desarrollo de una perspectiva distante de la oficial a la hora de encarar el conocimiento profundo de la realidad urbana, pero esta crítica nunca sobrepasa unos límites prefijados. El estudioso también busca contribuir a la mejora de su país y ejerce su autonomía para subrayar aquellas cosas que no funcionan. Esta perspectiva vuelve a ponerse de manifiesto en los análisis sobre la liberalización desigual del mercado del suelo y en los efectos de la transición económica china en la función residencial y comercial de las ciudades. Unas ciudades - y un volumen importante de trabajos insiste en este enfoque- que se han modernizado de forma acelerada, han crecido y han instalado las más modernas infraestructuras para una mayor eficiencia en su funcionamiento. La lógica del progreso urbano justificaba 22 trabajos, junto a los 9 referidos al sistema urbano, una cifra siempre superior a los que cuestionan los problemas ambientales generados por la urbanización (solo 4), si bien no alcanzan los 55 (más de la mitad del total) que hemos comentado, e insistían en la vivienda, el mercado del suelo, la segregación espacial y los grupos diferenciados que definen a la China metropolitana de comienzos del siglo XXI.

La revista Cities es la que presta mayor atención al fenómeno urbano chino de todas las analizadas. Le ha dedicado un total de 86 artículos sobre un total de los 880 publicados de 2005 a 2015, con un incremento progresivo de la atención a las ciudades de dicho país conforme nos acercamos a la actualidad. Como se puede deducir, el objetivo científico de esta publicación académica periódica es diferente al de Antipode y al de Urban Studies, ya que se centra en la política y en la planificación urbanas. Por este motivo, la temática de las contribuciones referidas a la República Popular China también es distinta, con una 
mayor diversificación temática. Así, si en Urban Studies dominaban los trabajos referidos a la segregación espacial, el mosaico urbano y el mercado inmobiliario, aquí se aprecia un mayor equilibrio entre el planeamiento, la gobernanza y la política de la ciudad, con 22 artículos; la cuestión de las desigualdades residenciales, el perfil ocupacional de los barrios y el uso del suelo, que es el tema de 17 escritos; la sosteniblidad y la ecología urbanas, que alcanzan los 16 textos, mientras que, para conocer el sistema de ciudades, los perfiles de algunas de ellas o el mercado del suelo disponemos de 12, 13 y 2 contribuciones respectivamente. Los restantes textos no son clasificables. Resulta lógico que, en un medio como Cities, interese la planificación urbana, el acercamiento a ejemplos individuales de ciudades chinas o los estudios sobre el sistema metropolitano, ya que son temas que encajan perfectamente con los objetivos de la revista. En cualquier caso, que sea la que más preocupación refleje por esta gran potencia también nos aclara que el extraordinario crecimiento y embellecimiento urbano asociado al éxito económico es un tema que no pasa desapercibido en prestigiosos medios científicos occidentales (con hegemonía estadounidense, en este caso) de la arquitectura, el urbanismo y la geografía de la ciudad. De hecho, que Cities consagre una serie al estudio de capitales provinciales o regionales chinas bajo el rótulo de profiles refleja una preocupación específica por disponer de datos para ejemplos urbanos donde consultoras de planeamiento, empresas de promoción o diseñadores urbanos occidentales puedan trabajar o colaborar con profesionales locales. Por su parte, en Cities, se dedica una atención muy superior a los aspectos ambientales metropolitanos, lo que posee un indudable interés, y, de nuevo, los contrastes residenciales, la complejidad social y la estructura de la ciudad contemporánea china vuelven a ser objeto de atención, incluso en una revista no especialmente centrada en estos temas.

Como apunte suplementario, hemos realizado esta misma consulta en las dos revistas españolas que figuran en el listado JCR - Boletín de la AGE y Scripta Nova-, y el resultado es muy negativo: se observa una casi total desatención de las mismas hacia China y su potencial, además de un olvido completo respecto a sus ciudades y espacios urbanos. De hecho, un repaso a los contenidos que publicaron entre 2005 y 2015, ambos incluidos, revela que solo se encuentran dos artículos acerca de dicho tema entre sus páginas, los cuales no son especialmente geográficos ni de carácter divulgativo. Se trata del elaborado por D. Gomá sobre la minoría coreana en China y publicado en Scripta Nova, aunque, por su contenido, no deje de ser un análisis desde la historia contemporánea a partir de fuentes secundarias (Gomá, 2008). También E. M. ${ }^{a}$ Martín Roda plantea un análisis sobre los desequilibrios territoriales en el auge económico chino, en un texto editado por el Boletín de la $A G E$, que recurre a datos macroeconómicos disponibles en series de amplia difusión internacional (Martín Roda, 2014).

En síntesis, este repaso a los trabajos publicados por las revistas globales al mundo urbano chino nos expresa un creciente interés hacia el mismo. Por supuesto, una atención especial por calibrar los impactos que las reformas económicas de los últimos decenios generan en unas sociedades ciudadanas 
muy complejas. Finalmente, la China del crecimiento (urbano, económico, monumental, etc.) también focaliza los estudios de los académicos occidentales, que, poco a poco, van descubriendo un mundo más diverso y policéntrico del que estimaban hasta hace pocos años.

\section{El contenido de este número}

Desde una perspectiva hispana o latina, como la que simboliza la revista Documents d'Anàlisi Geogràfica, que siempre ha cumplido un papel importante de divulgación y debate en la geografía española, la introducción de novedades científicas provenientes de otras regiones del mundo parece obligada. Así, nuestra progresiva curiosidad por lo que acontece en la geografía y en el análisis urbano chinos se ha combinado con un interés destacable de los geógrafos de ese país por publicar en castellano para el espacio hispano. En cualquier caso, nuestro contacto con los colegas de Pekín, Shanghai o Guangzhou a partir de 2003 mereció poca atención en el grupo de geografía urbana de la UGI, ya que sus aportaciones sonaban demasiado oficiales (el crecimiento de las ciudades, un sistema urbano de megalópolis, etc.), pautadas y no se comprendían demasiado bien en el inglés global empleado en los congresos.

Esta percepción cambió en el Congreso Regional de la UGI celebrado en Kioto en 2013, donde se nos encargó dirigir una sesión dominada por geógrafos de ese país, y nos sorprendimos al ver imágenes de personas sin techo y de infravivienda en Pekín, así como comunidades elitistas cerradas de millonarios en determinadas capitales regionales. Algo estaba pasando en la geografía urbana china que transmitía un cambio de temáticas y focos de interés. Una visita al país oriental durante ese mismo año nos ratificó esta idea de que algo se movía en la lectura académica del gigante asiático. Por esta razón, nos dirigimos a un viejo conocido de la UGI, el profesor Gu Chaolín, de la Universidad Tshingua de Pekín, a quien propusimos la idea de confeccionar este número. Gu ha utilizado la estructura del grupo de geografía urbana de la asociación de geógrafos chinos para contactar con varios colegas y aportarnos tres interesantes trabajos sobre los problemas sociales y residenciales de las metrópolis de Pekín y Guangzhou en la actualidad. El propio Gu, junto a dos de sus discípulos, nos muestra las pautas de asentamiento de "la tribu de las hormigas», antiguos estudiantes superiores de origen rural que no regresan a su aldea de origen y siguen viviendo alegalmente en la región de Pekín. Yanwei Cham y Yue Shen, de la Universidad de Pekín y de la Normal del Este de China, respectivamente, caracterizan los espacios suburbanos de Pekín como un complejo mosaico de poblaciones definidas a partir del paradigma de la movilidad. Por último, Xin Yin y Zhiang Li, de Guangzhou, realizan una original aproximación a las pautas residenciales de los emigrantes africanos en dicha gran metrópoli del gigante asiático meridional. Con estos tres textos, se pretende presentar una muestra de lo que elabora la geografía urbana china del presente, sus preocupaciones y sus puntos de vista críticos. Un enfoque que denuncia desajustes del sistema, 
bebe de los grandes analistas occidentales sobre la ciudad contemporánea, pero retratando el mundo chino como una unidad en sí, relevante, compleja y que necesita mejorar en su gestión; quizás como nos recordaba G. Davies por el bien del país que está llamado a convertirse en la primera potencia mundial del siglo XXI.

\section{Referencias bibliográficas}

Andreas, J. (2009). «Cambio de curso en China». New Left Review, 54 (enero-febrero), $117-137$.

Arrighi, G. (2007). Adam Smith en Pekin: Origenes y fundamentos del siglo XXI. Madrid: Akal.

BAilly, A. et al. (1984). Les concepts de la géographie humaine. París: Mason.

Benko, G. y Strohmayer, U. (ed.) (2004). Human Geography: A History for the 21st Century. Londres: Arnold Pbs.

Berman, M. (1982). Todo lo sólido se desvanece en el aire: La experiencia de la modernidad. Madrid: Siglo XXI.

Brunet, R.; Ferras, R. y Théry, H. (1992). Les Mots de la Géographie: Dictionaire critique. Montpellier: Reclus-La Documentation Française.

Capel, H. (1981). Filosofía y ciencia en la geografía contemporánea. Barcelona: Barcanova.

Chaunu, P. (1972). La expansión europea (siglos XIII al XV). Barcelona: Labor.

Davies, G. (2007). Worrying about China: The language of Chinese Critical Inquiry [en línea]. Cambridge (MA): Harvard University Press. <http://dx.doi.org/10.4159/9780674030237>

FarinelLi, F. (2003). Geografia: Un'introduzione ai modeli del mondo. Turín: Einaudi.

Friedmann, J. (2011). «Barrio por barrio: Reclamando nuestras ciudades». Urban: Los futuros de la planificación, 1 (marzo), 13-21. Madrid: Universidad Politécnica de Madrid. Departamento de Urbanística y Ordenación del Territorio.

Gomá, D. (2008). "La minoría coreana en China: Identidades y adaptación en un espacio geográfico fronterizo». Scripta Nova, 276 (1 de noviembre).

Gómez Mendoza, J. (2013). «Claves de la urbanización china». Revista de Obras Públicas, 3549 (diciembre), 21-30. Madrid: Colegio de Caminos, Canales y Puertos.

Gómez Mendoza, J. et al. (1982). El pensamiento geográfico. Madrid: Alianza.

Harvey, D. (2003). París, capital de la modernidad. Madrid: Akal.

Johnston, R.J.; Gregory, D. y Smith, D.M. (1987). Diccionario de Geografía Humana. Madrid: Alianza.

Levy, J. y Lussault, M. (dir.) (2003). Dictionnaire de la Géographie. París: Nelin.

Lladó, B. (2013). Franco Farinelli: Del mapa al laberinto. Barcelona: Icaria.

Martín Roda, E.M.a (2014). «Desequilibrios territoriales en el auge económico chino". Boletin de la AGE, 66, 143-160.

Mayhew, S. (1992). Dictionary of Geography. Oxford: Oxford University Press.

Menzies, G. (2003). 1421: El año en que China descubrió el mundo. Barcelona: Grijalbo.

Ortega Valcárcel, J. (2000). Los horizontes de la geografia: Teoría de la geografía. Barcelona: Ariel.

Roque de Oliveira, F. (2003). «Os portugueses e a Ásia marítima (1500-1640): Contributo para uma leitura global da primeira expansâo europeia no oriente». Scripta Nova, VII (151 y 152) (15 de octubre). 46 p. 
- (2007). «Una especie de invisibilidad: Limitaciones de la divulgación internacional de la literatura de los descubrimientos portugueses y el ejemplo del saber geográfico sobre la China». En: Navarro, V. y Eamon, W. (coords.). Más allá de la Leyenda Negra: España y la revolución científica. Valencia: Prensas Universitarias de Valencia, $105-120$.

Sassen, S. (2015). Expulsiones. Buenos Aires y Madrid: Katz.

Wallerstein, I. (1979-1998). El moderno sistema mundial I, II y III. México: Siglo XXI.

Zhaо, H. (2009). «Desvelarse por la nación». New Left Review, 54 (enero-febrero), $147-155$. 